\title{
Ciencia ciudadana y herramientas de comunicación en la Red Sismológica Nacional de la Universidad de Costa Rica
}

Recibido: $\quad 30$ de octubre, 2019.

Aceptado: 12 de octubre, 2020.

Por: Dr. Lepolt Linkimer Abarca ${ }^{1}$, Universidad de Costa Rica, Costa

Rica, ORCID: 0000-0002-1008-846X, y Dra. Ivonne Arroyo Hidalgo², Universidad de Costa Rica, Costa Rica, ORCID: 0000-0003-3232-9719.

\section{Resumen}

Costa Rica se ubica en el límite entre varias placas tectónicas, por lo que los sismos son comunes y parte de la vida cotidiana de sus habitantes. La Red Sismológica Nacional (RSN), con sede en la Universidad de Costa Rica (UCR), ha incursionado en la ciencia ciudadana empleando Tecnologías de la Información y la Comunicación para notificar y recolectar datos sobre los sismos. En este artículo, se describen diversos mecanismos usados por la RSN para difundir rápidamente información, creados en el marco de un proyecto de Acción Social inscrito en la UCR, que incluyen dos herramientas para el cálculo rápido de la intensidad sísmica llamadas Escenario de intensidades esperadas y ¿Lo sentiste? Estas herramientas están basadas en regresiones matemáticas y en el aporte voluntario de los ciudadanos, respectivamente.

Además, se presentan los resultados para el terremoto de Armuelles, de junio de 2019 (magnitud 6,4), construidos con una alta participación ciudadana. Los mapas de intensidades elaborados en pocos minutos por las dos herramientas concuerdan y muestran las zonas en donde el sismo provocó daños. La difusión de temas sismológicos por diferentes vías tiene un alto alcance. Especialmente, en redes sociales como Facebook, en donde una publicación puede alcanzar hasta 275 mil usuarios, lo cual demuestra su capacidad para informar y educar masivamente. El abordaje de la comunicación de la RSN apunta hacia un ambiente de colaboración y de participación social que tiene el potencial de impactar positivamente la percepción de la amenaza sísmica en Costa Rica.

1 El doctor Lepolt Linkimer Abarca es profesor catedrático de la Escuela Centroamericana de Geología y coordinador de la Red Sismológica Nacional, Universidad de Costa Rica, Costa Rica. Contacto: lepolt.linkimer@ucr.ac.cr.

2 La doctora Ivonne Arroyo Hidalgo es investigadora de la Red Sismológica Nacional, Universidad de Costa Rica, Costa Rica. Contacto: ivonne.arroyo@ucr.ac.cr.
Lepolt Linkimer Abarca e Ivonne Arroyo Hidalgo. Ciencia ciudadana y herramientas de comunicación en la Red Sismológica Nacional de la Universidad de Costa Rica. Revista Comunicación. Año 41, volumen 29, número 2 , julio-diciembre, 2020. Instituto Tecnológico de Costa Rica. ISSN: 0379-3974/e-ISSN1659-3820.

\section{PALABRAS CLAVE:}

sismo, educación ciudadana, tecnología de la información, redes sociales, Costa Rica, intensidad sísmica.

\section{KEY WORDS:}

earthquake, Citizenship education, information technology, social media, Costa Rica, seismic intensity. 


\section{Abstract}

\section{Citizen science and communication tools at the National Seismological Network of Costa Rica}

Costa Rica's location at the boundary of several tectonic plates makes earthquakes commonplace and part of daily life. The National Seismological Network (RSN) of the University of Costa Rica (UCR) has ventured into citizen science by utilizing Information and Communication Technologies to notify and collect earthquake data. This article describes different mechanisms implemented by the RSN to rapidly disseminate information. These mechanisms were created as part of an Outreach Project at UCR, which include two tools for the rapid calculation of seismic intensity called "scenario of expected intensities" and "Did you feel it?". These tools are based on mathematical regressions and the voluntary contribution of citizens, respectively.

We present results for the Armuelles earthquake in June 2019 (magnitude 6.4), which obtained high citizen participation. Intensity maps prepared in a few minutes by the two tools agree and show the areas where the earthquake caused damage. The dissemination of seismological topics by different means has a high reach, especially in social networks such as Facebook, where a publication can reach up to 275,000 users, demonstrating its ability to inform and educate massively. The RSN communication approach points to an environment of collaboration and social participation with the potential to positively impact the perception of seismic hazard in Costa Rica.

\section{INTRODUCCIÓN}

El concepto de ciencia ciudadana se define como la producción científica ejecutada por científicos y ciudadanos en conjunto, que está basada en la participación consciente y voluntaria de gente común, que genera datos a partir de sus interacciones sociales y con el medio ambiente (López, 2005; Gura, 2013). Con base en la tendencia mundial de participación de la sociedad civil en la recopilación, el intercambio y la difusión de datos con fines científicos (Gura, 2013), la Red Sismológica Nacional (RSN), con sede en la Universidad de Costa Rica (UCR), ha incursionado recientemente en la ciencia ciudadana a través del uso de la Tecnología de la Información y la Comunicación (TIC); en especial, a través de tecnologías móviles.

Costa Rica es uno de los países más sísmicos del planeta. Esta situación obedece a su ubicación en una zona de subducción en donde interactúan cuatro placas tectónicas: Coco, Caribe, Nazca y Panamá (figura 1). Desde 1821, el país ha sufrido el embate de 68 terremotos, 20 de los cuales han tenido magnitud mayor a 7,0 (Montero, 1989; Peraldo y Montero, 1994; Linkimer y Alvarado, 2014). Entre los terremotos más recientes, se pueden contar el de Cinchona en el 2009 (magnitud 6,1) y el de Nicoya en el $2012(7,6)$. La RSN ha registrado, desde 1975, alrededor de 90 sismos con magnitud mayor a 5,5 y 34 de estos han ocasionado víctimas o algún tipo de daño en edificaciones (figura 1). Desafortunadamente, al menos 456 personas han perdido la vida durante los terremotos, la mayoría ( 300) durante la destrucción de Cartago en el terremoto de 1910 (Montero y Miyamura, 1981; Montero, 2010).

La RSN es un programa de investigación de la UCR y es uno de los entes dedicados a la vigilancia sísmica del país. Tiene su sede en la Escuela Centroamericana de Geología de la UCR desde 1982 y sus raíces se encuentran en los estudios sismológicos llevados a cabo por la UCR y el Instituto Costarricense de Electricidad (ICE) desde los años 70 (Linkimer, Arroyo, Alvarado, Arroyo y Bakkar, 2018). La estrecha colaboración entre los investigadores del ICE y la UCR ha promovido el intercambio de datos entre ambas instituciones y ha permitido la formalización de convenios de cooperación científica y técnica vigentes hasta la actualidad. 
Como resultado, la RSN opera, en el 2020, una red de 167 estaciones sismológicas que transmiten en tiempo real, de las cuales 118 son administradas por la UCR y el resto (49) por el ICE (figura 1). Esta red de instrumentos permite generar gran parte de la información sismológica que se difunde con los mecanismos descritos en este artículo. El objetivo de la RSN es desarrollar conocimiento científico sobre la geodinámica interna de la Tierra, para transferirlo a la sociedad costarricense a través de la docencia y de la acción social. En Costa Rica, existe, además, el Observatorio Vulcanológico y Sismológico de Costa Rica (Ovsicori) de la Universidad Nacional y el Laboratorio de Ingeniería Sísmica de la UCR. Ambos centros funcionan desde 1984 (Morales, 1986) y también investigan la sismicidad y sus efectos.

Aunque en Costa Rica los sismos son parte de la vida cotidiana de sus habitantes, los programas formales de educación y los medios de comunicación masiva aún no han implementado una forma apropiada de educar a la población en este tema, por lo que persisten mitos y confusiones. Esta situación es uno de los aspectos que motivaron la formulación de un proyecto de Acción Social (ED-3005) inscrito en la UCR desde el 2013, titulado Difusión de temas sismológicos.

El objetivo de este proyecto ha sido divulgar información relacionada con los sismos a través de diversos mecanismos de comunicación, con el fin de poner a disposición de la mayor cantidad de personas la información científica generada por la RSN y con esto incidir en la reacción y percepción que tienen los habitantes de Costa Rica ante los fenómenos sísmicos. Entre los mecanismos de comunicación usados están: una página web, redes sociales (Facebook, Twitter, Instagram y YouTube), una aplicación gratuita para teléfonos inteligentes, las charlas en comunidades, las visitas guiadas a la sede de la RSN en la UCR y la publicación de artículos científicos, de panfletos, de videos y de material educativo.

En este artículo, se describen algunos de los mecanismos creados en el marco del mencionado proyecto de Acción Social y se da énfasis a las herramientas Ilamadas: escenario de intensidades esperadas y ¿Lo sentiste? Esta última se encuentra disponible para teléfonos móviles y permite el cál- culo de intensidades con base en la colaboración ciudadana. Dicha aplicación es relevante pues utiliza la TIC en la recopilación de información sismológica y, además, es la única de su tipo funcionando actualmente en Costa Rica. Asimismo, se presenta la metodología usada para la determinación de la intensidad y se incluyen resultados obtenidos para algunos sismos recientes.

Inevitablemente, por su contexto tectónico (figura 1), Costa Rica continuará experimentando sismos y terremotos, por lo que la preparación para afrontar este fenómeno es fundamental. Esta preparación no se alcanza de la noche a la mañana, pero comienza cuando la población comprende y se familiariza con los sismos. Por tanto, ese es el norte de los esfuerzos en comunicación que aquí se documentan.

Figura 1: Contexto tectónico de Costa Rica y el mapa de estaciones sismológicas de la RSN durante el 2020.

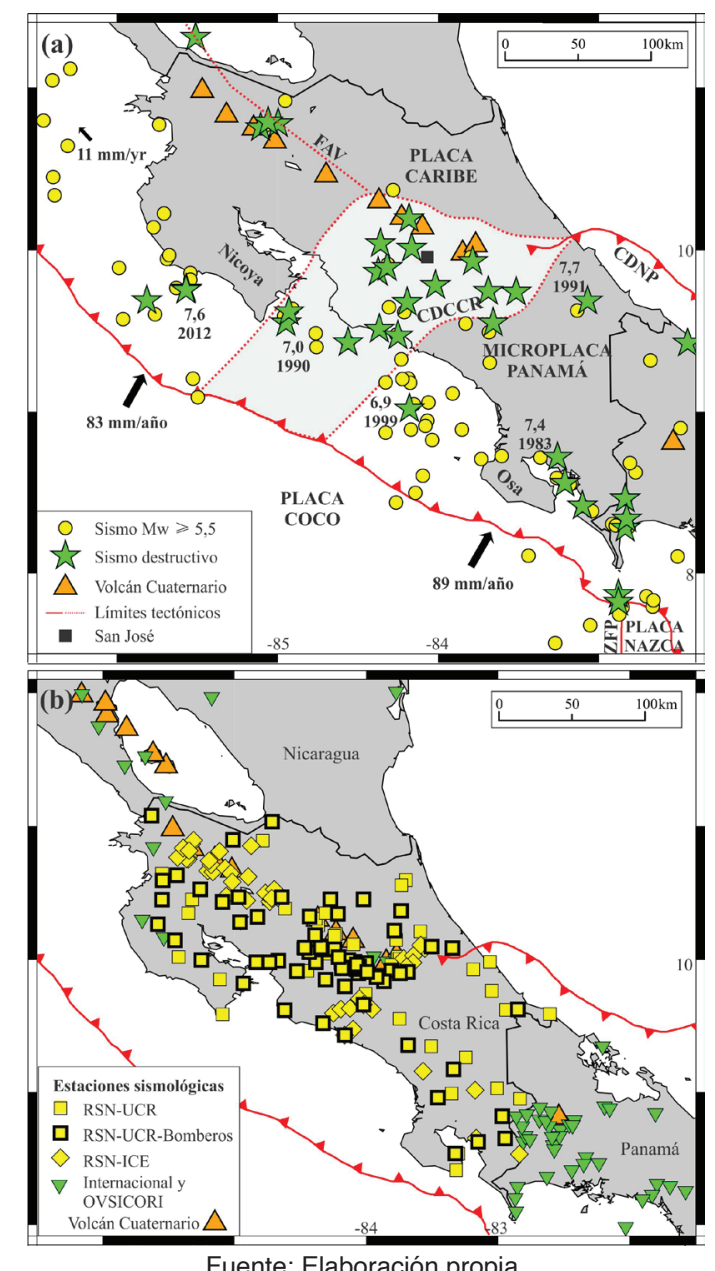


En la figura 1, la parte (a) representa el contexto tectónico de Costa Rica. Las líneas rojas representan los principales límites tectónicos, como el Cinturón Deformado del Centro de Costa Rica (CDCCR), el Cinturón Deformado del Norte de Panamá (CDNP), las Fallas del Arco Volcánico de Guanacaste (FAV) y la Zona de Fractura de Panamá (ZFP). La parte (b) representa el mapa de estaciones sismológicas de la RSN durante el 2020.

\section{MARCO TEÓRICO}

Los conceptos de intensidad sísmica y de la práctica de participación pública Ilamada ciencia ciudadana forman el núcleo teórico del presente artículo. La intensidad sísmica es una medida del grado de vibración del terreno y de los efectos y daños causados por un sismo. La forma clásica de determinar la intensidad consiste en la observación de los efectos producidos en las construcciones y el impacto que provoca en las personas. El conjunto de observaciones en cada localidad se asocia, entonces, con una descripción predefinida a modo de escala. En el mundo, existen diferentes tipos de escalas de intensidad, pero en Costa Rica la más empleada es la Mercalli Modificada (IMM). En ella, los reportes de intensidades se agrupan en doce grados, expresados usualmente en números romanos (Wood y Newman, 1931). Al respecto, del I al VI cada grado corresponde con la apreciación del movimiento (¿cómo lo sintió?); del VII al X con la severidad de los daños; del X al XII con los cambios geológicos producidos.

La intensidad, también, se puede determinar a partir de relaciones matemáticas entre el parámetro mencionado y algunas variables que lo afectan; por ejemplo: distancia y magnitud. Además, a partir de mediciones instrumentales de la aceleración del suelo que pueden ser convertidas a intensidad con base en regresiones matemáticas (Linkimer, 2008a). Este último tipo de cálculo, no es abordado rutinariamente por la RSN y, por eso, queda fuera del alcance de este artículo.

La magnitud es distinta a la intensidad, ya que es una medida instrumental del tamaño del temblor y se designa con un número que no tiene unidades ni límites y es un valor único para cada sismo. La intensidad, en cambio, varía de un lugar a otro para un mismo sismo y depende de muchos factores, entre ellos: la magnitud y duración del sismo, la distancia al epicentro, la profundidad del hipocentro, la geología y la topografía local que podrían inducir la amplificación de las ondas sísmicas, y aspectos relacionados con el observador, que van desde su ubicación en campo abierto o en un edificio alto, hasta su temperamento. Todo esto, hace que la intensidad sea de cierta manera subjetiva, a diferencia de las medidas instrumentales de la aceleración del terreno (Gutenberg y Richter, 1942; Gutenberg y Richter 1956; Wald, Quintoriano, Heaton y Kanamori, 1999; Linkimer, 2008a).

A pesar de la relativa subjetividad de las intensidades recopiladas, estos valores son muy útiles para la interpretación de los sismos históricos, ya que no cuentan con registros instrumentales. Asimismo, los mapas de intensidades de sismos actuales son importantes pues permiten analizar cuáles zonas podrían haber sido las más afectadas por un terremoto y cuánta población pudo haber estado expuesta a un determinado grado de intensidad. Estos mapas posibilitan, además, detectar patrones de atenuación regionales y también 'efectos de sitio'. Es decir, las zonas particulares en donde se experimenta una mayor intensidad, que luego pueden ser estudiadas a fondo para determinar el origen de esa amplificación (Wald et al., 1999; Atkinson y Wald, 2007).

Para crear mapas de intensidades confiables, es necesario recabar la información de un número elevado de observadores y desde muchas localidades (Linkimer, 2008b). Con este fin, la sismología se ha intersectado recientemente con la ciencia ciudadana a través de la comunicación digital (Bossu et al., 2018). A nivel mundial, actualmente existen una gran variedad de proyectos científicos basados en la recopilación de datos de forma consciente y voluntaria por parte de ciudadanos, que incluyen además de las observaciones sismológicas, las climatológicas, astronómicas y biológicas, entre muchas otras (López, 2005; Gura, 2013; Asorey et al., 2017; Bossu et al., 2018; Finazzi, 2020). La ciencia ciudadana permite a los científicos obtener los datos que necesitan y, al mismo tiempo, involucrar a la población en resolver problemas de alta pertinencia social. En la mayoría de los casos, cualquier persona puede 
contribuir, ya sea con su experiencia, conocimiento o recursos tecnológicos y según su disposición y voluntad para trabajar colaborativamente.

En ciencia ciudadana, es vital la educación de las personas involucradas y es muy común que los proyectos educativos estén liderados por universidades (Gura, 2013). Un ejemplo es la red ambiental ciudadana de monitoreo desarrollada por la Universidad Industrial de Santander en Colombia. En este proyecto, se han capacitado estudiantes y maestros para la construcción, el uso y el mantenimiento de estaciones meteorológicas fabricadas con bajo costo y que miden diversas variables climáticas y de contaminación del aire (Asorey et al., 2017).

En sismología, la comunicación digital ha permitido ofrecer información rápida a las zonas afectadas por sismos sentidos $y$, al mismo tiempo, recolectar las observaciones aportadas por los testigos de tales eventos (Allen, 2012; Bossu et al., 2018; Finazzi, 2020). El extenso uso de dispositivos móviles y de redes sociales ha permitido, incluso, observar la manera en la que los mensajes publicados en Twitter relacionados con un temblor se expanden en el tiempo y el espacio, como si fueran una onda similar a la de la sacudida (Allen, 2012; Crooks, Croitoru, Stefanidis, y Radzikowski, 2013).

En este sentido, la estrategia de aprovechar las redes sociales y las aplicaciones para teléfonos móviles ha sido aplicada exitosamente por el Servicio Geológico de los Estados Unidos y el Centro Sismológico Europeo Mediterráneo (Allen, 2012; Bossu et al., 2018). Esto debido a que brindar información rápida y oportuna ha fomentado el compromiso de la población en el reporte de intensidades (Bossu et al., 2018) y, además, ha sido beneficioso en la respuesta de la población a la amenaza de tsunamis (Blake et al., 2018).

\section{METODOLOGÍA}

La metodología que se sigue en la RSN para el cálculo de intensidades con las herramientas escenario de intensidades esperadas y ¿Lo sentiste? inicia con el procesamiento rutinario de la localización y magnitud del temblor. En este primer paso, se generan datos preliminares a partir de los programas de detección y localización automática del sistema SeisComP3 (Gempa, 2019). Los resultados son publicados en dos o tres minutos de forma automática en las redes sociales Facebook y Twitter, y en la aplicación para teléfonos móviles RSN. Esta publicación incluye únicamente la fecha, la hora, la localización epicentral y la magnitud del sismo. A partir de la publicación de esta información preliminar, los usuarios con acceso a los medios mencionados, comienzan a brindar reportes del temblor, como se detalla más adelante.

El segundo paso consiste en la revisión de la localización preliminar por parte de un analista entrenado en sismología y en la publicación de la localización revisada, entre 5 y 20 minutos después del tiempo de origen del sismo. Este análisis de los sismogramas, la localización y el cálculo de la magnitud momento (Mw) se llevan a cabo usando los programas integrados en el software sismológico Seisan (Ottemöller, Voss y Havskov, 2019). El resultado de este segundo paso es confirmar y corregir, si es necesario, los datos publicados inicialmente. La publicación de cada sismo revisado se realiza en la página web, en la aplicación móvil y en las redes sociales mencionadas previamente. Además, esta publicación incluye un mapa del epicentro y las características básicas del temblor, tales como: la fecha, la hora, la localización, la magnitud y la profundidad.

En este punto, se realiza, en conjunto con la localización revisada, la estimación de la intensidad, la cual se publica en la página web como el escenario de intensidades esperadas (figura 2). Este mapa de intensidades se construye a partir de una relación teórica del decaimiento de la intensidad en la escala IMM con la distancia (R) y la magnitud (M). Esta relación fue determinada para Costa Rica a partir de observaciones de la intensidad de sismos históricos (Porras, 2017) y tiene la siguiente forma:

$\mathrm{IMM}=2,07+1,538 * \mathrm{M}+0,0016 * \mathrm{R}-3,952 * \log _{10} \mathrm{R}$ 
Figura 2: Escenario de intensidades esperadas para el terremoto de Armuelles del 25 de junio de 2019 (magnitud 6,4)

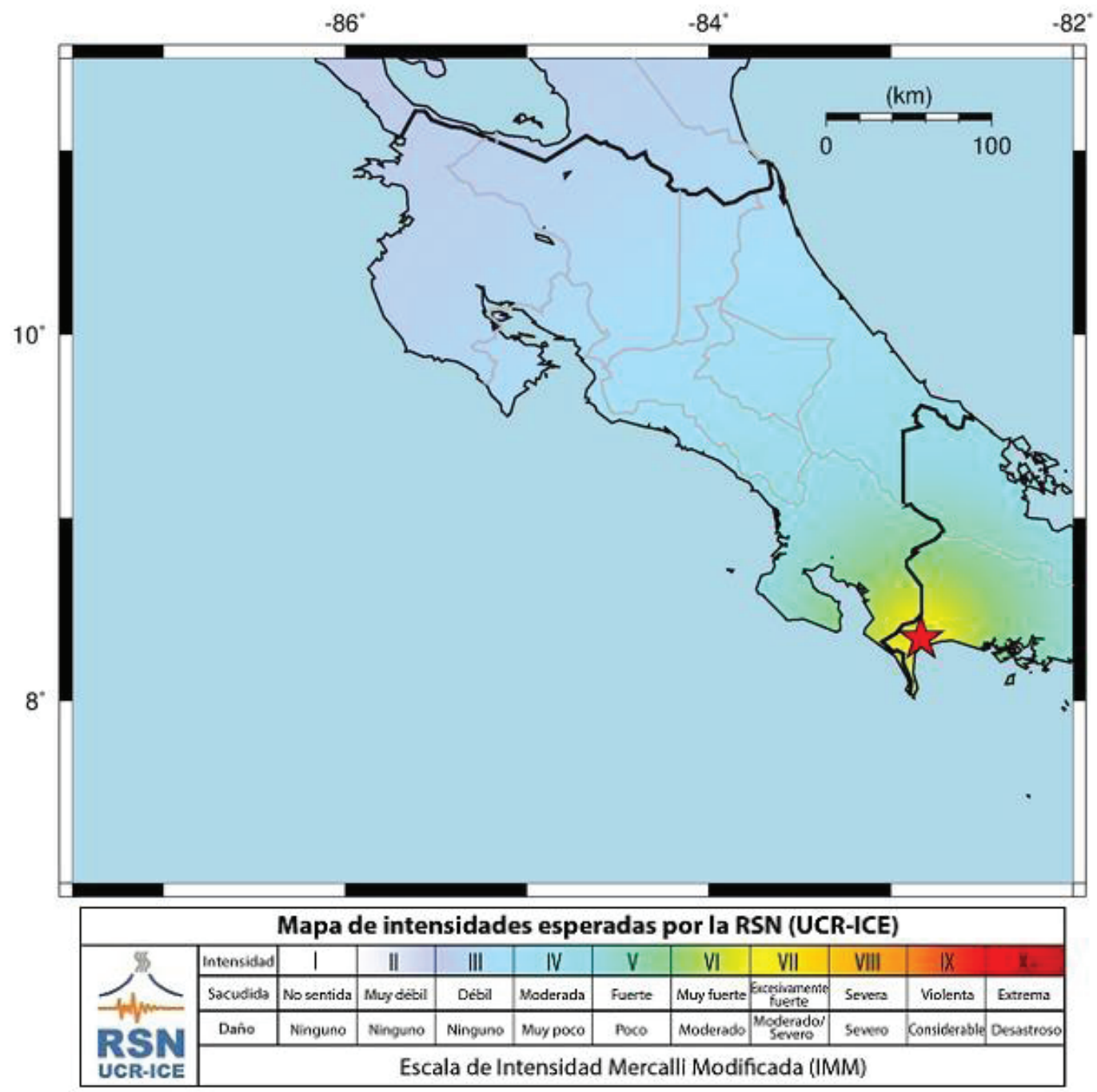

Fuente: Elaboración propia.

El escenario de intensidades esperadas sirve como referencia de comparación para los mapas de intensidades generados a partir de las observaciones de los usuarios. Estos mapas, se crean a partir del módulo ¿Lo sentiste?, disponible en la página web de la RSN y en la aplicación móvil (figura 3). Al respecto, el módulo ¿Lo sentiste? ha permitido el aporte de un vasto número de usuarios de internet con teléfonos inteligentes. Como se mencionó anterior- mente, los usuarios pueden comenzar a reportar un sismo a partir del momento en que se publica una localización preliminar (en el paso uno) y pueden continuar haciendo sus reportes durante la revisión y la actualización de la localización en todas las plataformas (posterior al paso dos). 
Figura 3: Aspecto del módulo ¿Lo sentiste? incluido en la aplicación para teléfonos móviles RSN

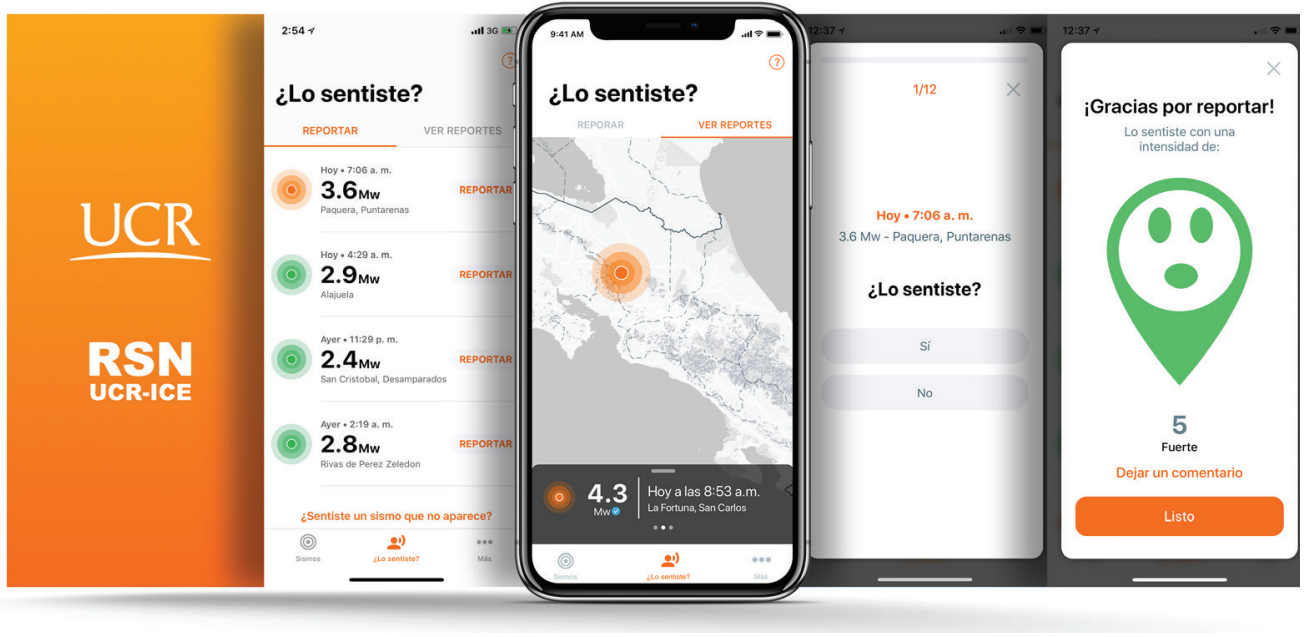

Fuente: Elaboración propia.

El mecanismo que se sigue para recibir y procesar la información aportada por los ciudadanos con el módulo ¿Lo sentiste? se detalla a continuación. La intensidad se calcula a partir de un cuestionario de 12 preguntas, inspirado en el que usa el Servicio Geológico de los Estados Unidos (Atkinson y Wald, 2007; Wald, Quitoriano, Worden, Hopper y Dewey, 2011), pero traducido al español y simplificado.

Para cada pregunta, el usuario escoge entre una serie de opciones predeterminadas. Las preguntas se responden en aproximadamente un minuto y son las siguientes: ¿lo sentiste?, ¿qué estabas haciendo?, ¿en qué lugar estabas?, ¿personas cerca tuyo lo sintieron?, ¿cómo describirías el movimiento?, ¿cómo reaccionaste?, ¿fue difícil caminar o mantenerse en pie?, ¿los objetos livianos se desplazaron o cayeron de los estantes?, ¿los cuadros se cayeron?, ¿los muebles se volcaron o cayeron?, y ¿hubo algún daño en las edificaciones?. Adicionalmente, el usuario tiene la opción de enviar comentarios para reportar si hubo algún efecto en la naturaleza, como deslizamientos, grietas en el suelo, entre otros.

Aunado a lo anterior, se utilizó el concepto de la intensidad decimal comunitaria (CDI por sus siglas en inglés), definida por Dengler y Dewey (1998) como un agregado de las sumas ponderadas (CWS) de los diversos índices asociados con las preguntas mencionadas anteriormente. El valor de la intensidad se determina con la ecuación (Wald, Quitoriano, Worden, Hopper y Dewey, 2011):

$$
\mathrm{CDI}=3,40 * \operatorname{lnCWS}-4,38
$$

Al final del cuestionario, el usuario recibe el valor de intensidad que se calculó de acuerdo con la combinación de sus respuestas y se genera un emoticono que representa la intensidad con que la persona sintió el sismo del 1 al 10 (figuras 3 y 4). Los emoticonos van desde el "no sentido" (intensidad 1) al "extremo" (10), pasando por las intensidades "muy débil" (2), "débil" (3), "moderado" (4), "fuer-

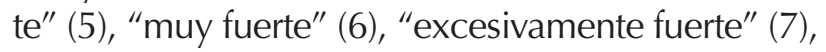
"severo" (8) y "violento" (9). Se decidió excluir los casos de intensidades más altas (11 y 12), pues son poco frecuentes y se determinan principalmente mediante la observación de cambios geológicos, que son más complejos de documentar.

Luego de generar el valor de intensidad, la aplicación envía automáticamente a los servidores de la RSN este cálculo, así como la ubicación geográfica en donde el usuario dijo haber sentido el sismo. Paralelamente, se reflejan en un mapa los emoticonos que representan los diferentes grados de intensidad (figura 4). Esta representación pretende hacer más amigable el contenido científico, y está inspirada en la tendencia dentro de la ciencia ciudadana, de convertir las tareas en juegos (Gura, 2013). El mapa con emoticonos puede ser accedido en tiempo real en la misma aplicación móvil o en la página web y va cambiando conforme más usuarios envían sus reportes. 
Figura 4: Mapa de reportes de intensidad a través del módulo ¿Lo sentiste? para el terremoto de Armuelles del 25 de junio de 2019 (magnitud 6,4)

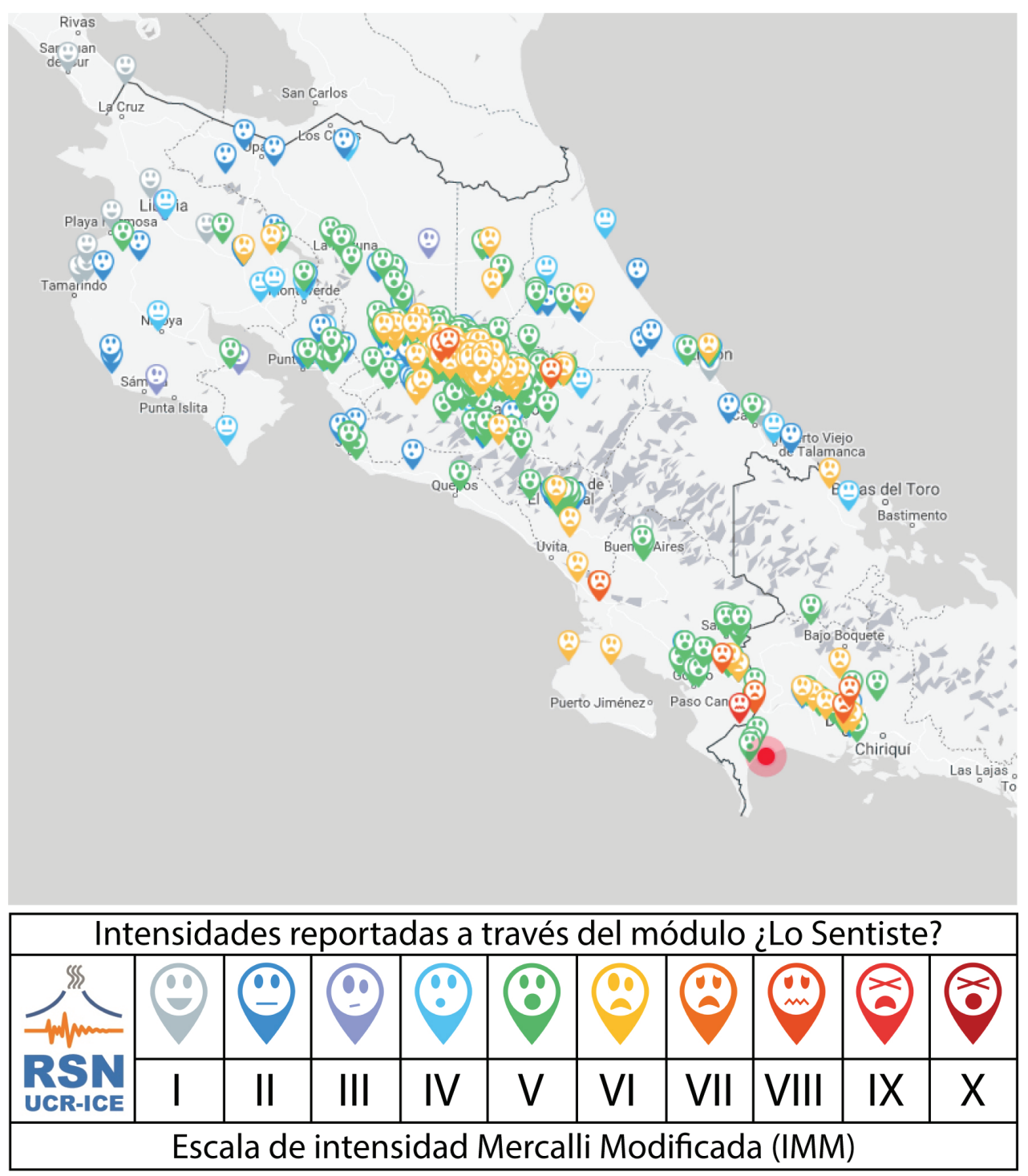

Fuente: Elaboración propia.

Durante el proceso de recolección de datos de intensidades, se realiza un control de calidad con el fin de descartar posibles datos erróneos, que pudieron haber sido aportados por usuarios inescrupulosos. A través de una inspección visual con criterio de experto, se localizan los valores de intensidad anómalos, que son los que muestran un valor muy alto en comparación con el resto de valores reportados alrededor. Los valores anómalos son poco frecuentes, se presentan, usualmente, uno o dos por sismo. Una vez identificados, son eliminados manualmente de la base de datos.
Posterior a la eliminación de los datos anómalos, con toda la información recolectada, se elabora el mapa de intensidad promediado, en el cual se presentan los valores de intensidad en celdas cuadradas de $5 \mathrm{~km}$ de lado. Cada celda se colorea de acuerdo con el valor de intensidad correspondiente (figura 5). Al igual que el mapa de emoticonos, el mapa promediado puede ser accedido desde la página web y desde los dispositivos móviles, usualmente, después de 10-30 minutos de haber ocurrido el temblor, cuando se han recopilado suficientes datos de intensidad. El mapa continúa actualizán- 
dose conforme más datos se adquieren durante las primeras 24 horas después de un sismo fuerte. Adicionalmente, tanto el mapa de emoticonos como el mapa promediado son divulgados en las redes sociales de la RSN.

Figura 5: Mapa promediado de intensidades para el terremoto de Armuelles del 25 de junio de 2019 (magnitud 6,4)
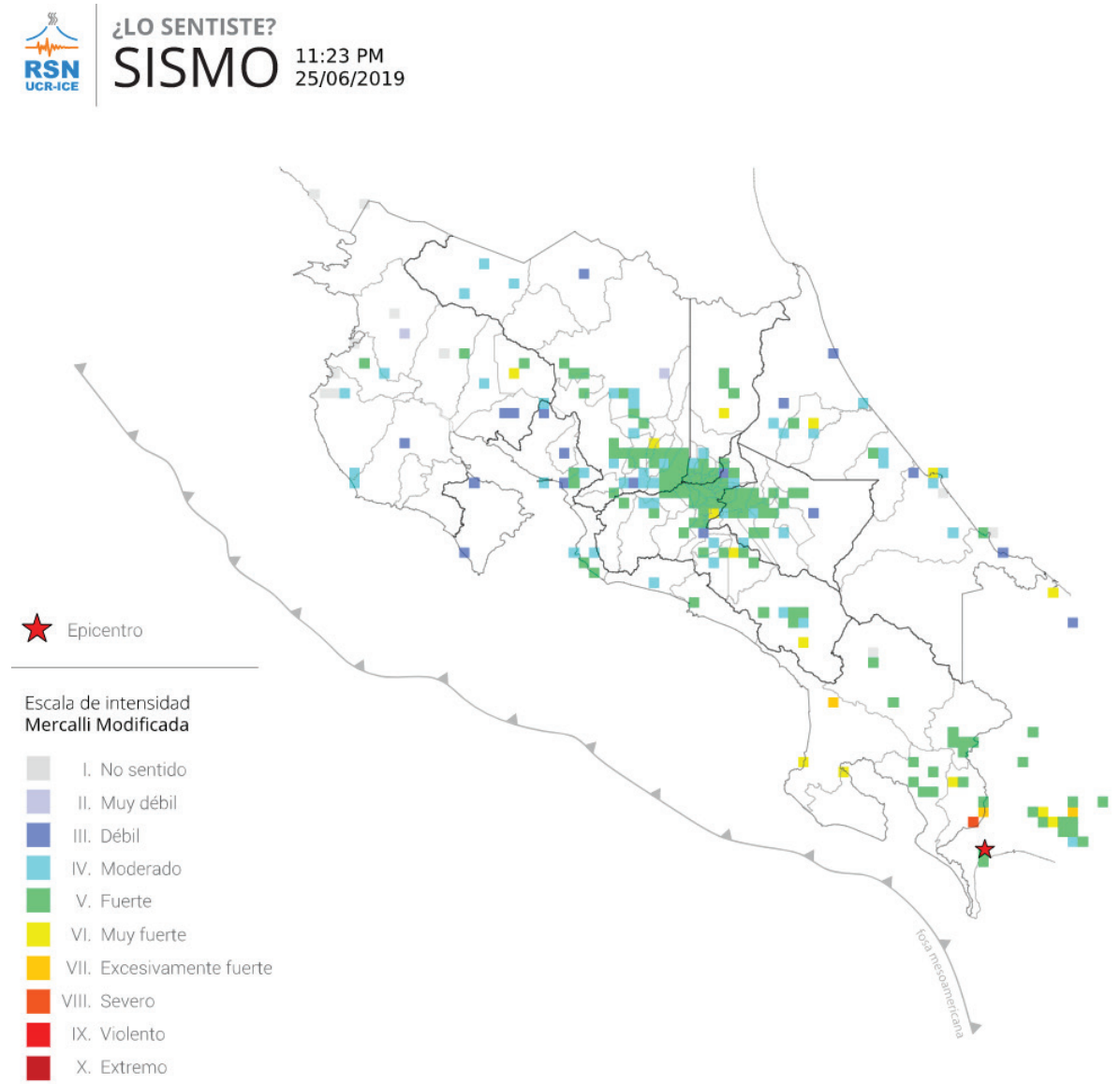

Fuente: Elaboración propia.

Todo el procesamiento de datos y la creación de los mapas mencionados anteriormente se realizan en los servidores ubicados en el Centro de Informática de la UCR; los cuales funcionan las 24 horas del día y todos los días del año para emitir localizaciones preliminares. Además, son accedidos remotamente por el personal de la RSN cada vez que ocurre un temblor fuerte, con el fin de publicar las localizaciones revisadas y mapas de intensidades.

\section{RESULTADOS}

Actualmente, la RSN cuenta con una página web (www.rsn.ucr.ac.cr), una aplicación para teléfonos inteligentes llamada $R S N$, y cuentas en las redes sociales Facebook (www.facebook.com/RSN.CR), Twitter (www.twitter.com/RSNcostarica), Instagram (www.instagram.com/rsn.cr) y YouTube (https:// www.youtube.com/user/RSNCostaRica). Todos estos medios son usados para difundir los resultados de las herramientas creadas para el cálculo rápido de la intensidad sísmica llamadas escenario de intensidades esperadas y ¿ Lo sentiste?

Estas herramientas han agilizado el acceso y la difusión de información sísmica a la población mediante el uso de la TIC. El mapa denominado escenario de intensidades esperadas se produce de forma automática con base en relaciones teóricas 
del decaimiento de la intensidad con la distancia, la magnitud y la profundidad del evento. El módulo llamado ¿Lo sentiste?, por su parte, disponible en la página web y la aplicación móvil $R S N$, ha permitido la construcción de mapas de intensidades, pero basados en las observaciones aportadas por los ciudadanos. Los detalles de estas dos herramientas se explicaron en la sección de metodología.

La aplicación RSN, gratuita y disponible para iOS y Android en las tiendas virtuales de la UCR, ha sido descargada unas 15 mil veces entre su lanzamiento en mayo de 2018 y julio de 2020. Los usuarios pueden revisar sismos recientes con su magnitud y su ubicación, ver un mapa con todos los sismos registrados en las últimas 24 horas o 15 días y acceder a los mapas de intensidades de los sismos más relevantes. Se debe mencionar que muy pocos países en el mundo cuentan con una aplicación móvil que posea un módulo como ¿Lo sentiste? El Ovsicori y países como México y Colombia poseen herramientas similares en sus páginas web; sin embargo, el proceso de reporte es complicado y difícil de visualizar. En cambio, en el caso de ¿Lo sentiste?, la herramienta se enfoca en facilitar el proceso de reporte de manera ágil y sencilla.
Entre mayo de 2018 y julio de 2020, 17 sismos han tenido más de 500 reportes de usuarios (tabla 1). Tres eventos, en particular, han tenido más de mil reportes: el terremoto de Golfito del 17 de agosto de 2018 (magnitud 6,1), el terremoto de Armuelles del 25 de junio de 2019 (magnitud 6,4) y el sismo de Boruca de Buenos Aires del 6 de marzo de 2020 (magnitud 5,6).

La lista de sismos con mayor número de reportes (tabla 1) incluye los eventos de mayor magnitud del período de tiempo analizado (Porras et al., 2019; Arroyo, Linkimer y Arroyo, 2020) y, además, algunos eventos que han tenido magnitud baja, pero cuyo epicentro se ubicó en la zona urbana de San José o en sus proximidades. La magnitud de los sismos mayores varía entre 5,1 y 6,4. De los sismos de menor magnitud, pero ubicados cerca de San José, sobresalen dos temblores de magnitud 3,9 y 4,5 ocurridos en Aserrí el 27 de julio de 2018, y un sismo en Coronado el 22 de noviembre de 2018, con magnitud 4,1. Estos eventos fueron reportados por una cantidad de entre 546 y 937 personas; especialmente, en la zona central de Costa Rica.

Tabla 1: Sismos con más reportes en el módulo ¿Lo sentiste? entre mayo de 2018 y julio de 2020

\begin{tabular}{|l|c|l|c|}
\hline \multicolumn{1}{|c|}{ Fecha y hora } & Mag. & \multicolumn{1}{c|}{ Localización } & Reportes \\
\hline Julio 27, 2018, 9:33 a.m. & 3,9 & $2 \mathrm{~km}$ al este de San Gabriel de Aserrí & 937 \\
\hline Julio 27, 2018, 11:50 a.m. & 4,5 & $1 \mathrm{~km}$ al sureste de San Gabriel de Aserrí & 833 \\
\hline Ago. 17, 2018, 5:22 p.m. & 6,1 & $8 \mathrm{~km}$ al suroeste de Golfito & 1044 \\
\hline Nov. 9, 2018, 3:20 a.m. & 5,9 & $9 \mathrm{~km}$ al noreste de Puerto Jiménez & 837 \\
\hline Nov. 17, 2018, 8:12 a.m. & 5,1 & $12 \mathrm{~km}$ al oeste de Guápiles & 706 \\
\hline Nov. 22, 2018, 10:39 p.m. & 4,1 & $1 \mathrm{~km}$ al noreste de Coronado & 546 \\
\hline Mayo 12, 2019, 1:24 p.m. & 6,0 & $5 \mathrm{~km}$ al sur de La Cuesta de Corredores & 686 \\
\hline Mayo 12, 2019, 7:01 p.m. & 4,8 & $15 \mathrm{~km}$ al norte de Parrita & 712 \\
\hline Junio 25, 2019, 11:23 p.m. & 6,4 & $11 \mathrm{~km}$ al este de Armuelles, Panamá & 1298 \\
\hline Ago. 6, 2019, 3:14 p.m. & 5,4 & $2 \mathrm{~km}$ al norte de Venecia de San Carlos & 671 \\
\hline Sep. 17, 2019, 10:58 p.m. & 5,3 & $13 \mathrm{~km}$ al norte de Cahuita & 689 \\
\hline Sep. 18, 2019, 5:23 a.m. & 5,2 & $18 \mathrm{~km}$ al oeste de Savegre de Quepos & 821 \\
\hline Nov 24, 2020, 7:33 p.m. & 5,6 & $10 \mathrm{~km}$ al noreste de Arancibia, Puntarenas & 733 \\
\hline Enero 21, 2020, 3:33 a.m. & 5.6 & $42 \mathrm{~km}$ al suroeste de Dominical, Osa & 534 \\
\hline Marzo 6, 2020, 8:40 p.m. & 5,6 & $14 \mathrm{~km}$ al este de Boruca de Buenos Aires & 1006 \\
\hline Abril 17, 2020, 3:34 a.m. & 4,6 & $13 \mathrm{~km}$ al este de Quepos & 576 \\
\hline Julio 5, 2020, 3:39 a,m. & 4,8 & $2 \mathrm{~km}$ al sur de Grifo Alto, Puriscal & 985 \\
\hline
\end{tabular}

Fuente: Elaboración propia. 
El sismo con más reportes hasta la fecha es, a su vez, el de mayor magnitud $(6,4)$. Este evento cerca de Armuelles, Panamá, fue reportado por casi 1300 personas (figuras 4 y 5), ubicadas en todas las provincias de Costa Rica y, también, en el occidente de Panamá. Afortunadamente, este terremoto no ocasionó pérdida de vidas humanas ni heridos, pero sí se reportaron daños de viviendas tanto en Pérez Zeledón, Osa, Corredores y Palmar Norte, como en Divalá y Gualaca, ubicadas en Panamá. También, hubo pérdidas económicas en abarrotes de centros comerciales de la Zona Sur de Costa Rica y oeste de Panamá. Además, se reportó la suspensión del servicio eléctrico en Palmar Sur, Pérez Zeledón, Ciudad Cortés, Puerto Jiménez y Parrita, y la caída del tendido eléctrico en Corredores.

Un aspecto relevante de documentar, es que la comparación entre el escenario de intensidades esperadas calculado automáticamente (figura 2) y el mapa promediado construido con los reportes de los usuarios (figura 5) muestra las mismas tendencias. Se puede observar que el sismo fue sentido en todo el territorio costarricense, con intensidades de hasta VI y VII en la escala Mercalli Modificada en Paso Canoas, Golfito y Laurel, y en Puerto Armuelles, David y pueblos cercanos en Panamá. En el Valle Central, el Pacífico Central y la costa Caribe Sur, se sintió con una intensidad de IV a V y muy levemente en gran parte de Guanacaste (figuras 2, 4 y 5$)$.

Otro aspecto notable, es que el módulo ¿Lo sentiste? fue muy usado en el occidente de Panamá, desde donde se recibieron reportes de 21 localidades en las provincias de Chiriquí y Bocas del Toro (figura 4). Esto permitió completar el mapa de intensidades más allá de las fronteras costarricenses (figura 5).

Al igual que con el terremoto de Armuelles, los mapas de intensidades generados por las dos herramientas para el resto de sismos con alta participación (tabla 1) fueron muy similares entre sí, lo cual indica una buena correlación entre las intensidades esperadas teóricamente y los reportes de los usuarios.

Todos los sismos mostrados en la Tabla 1, tuvieron también un alto impacto en las redes sociales, particularmente en Facebook. Se debe mencionar que el terremoto de Armuelles fue el que tuvo la más alta interacción. Las publicaciones de la localización automática y revisada Ilegaron a 275 mil y 106 mil personas, respectivamente; en conjunto, con una publicación inicial sobre la ocurrencia de este sismo, las tres publicaciones generaron alrededor de 9 200 comentarios.

\section{DISCUSIÓN}

El impacto de la difusión de la información generada por la RSN se puede analizar a través de dos aspectos: la rapidez para hacer disponible la información por diferentes vías y el gran número de personas alcanzadas con datos sismológicos y con material educativo a través de una variedad de medios y herramientas.

\section{Rapidez en la generación y difusión de informa- ción sismológica}

Actualmente, la población tiene acceso a los datos de un sismo sentido en tan solo 2-3 minutos a partir de su ocurrencia. Asimismo, los ciudadanos pueden acceder al escenario de intensidades esperadas en unos 5-20 minutos después del evento y al mapa promediado de intensidades de ¿Lo sentiste? en unos 10-30 minutos.

En el pasado, la difusión de la información por parte de la RSN era sumamente lenta, por lo que la población debía esperar varias horas e, incluso, días para conocer los detalles de un sismo sentido. La RSN inició la divulgación de información de sismos sentidos en julio de 1976. En ese entonces, la localización de los sismos se realizaba a través de un tedioso proceso, que requería utilizar el uso de una única computadora ubicada en el Centro de Informática de la UCR. Una vez que el sismo era localizado, se procedía a generar un boletín con la información, que luego era repartido por un mensajero en cada medio de comunicación ubicado en San José, o bien, se difundía por vía telefónica.

Los procedimientos para la localización y difusión de información fueron cambiando con la aparición del internet y otros recursos de comunicación. Hacia el año 2000, la RSN solía utilizar una máquina de fax para enviar los informes. No obstante, debido a que esto se realizaba individualmente para cada medio de comunicación y para la Comisión 
Nacional de Prevención de Riesgos y Atención de Emergencias (CNE), el proceso era aún ineficiente y podía demorar varias horas.

Con la creación de la primera página web de la RSN en el 2003, se comenzó a publicar la información de sismos sentidos más rápidamente. Paralelamente, se inició el envío de información por medio de correo electrónico a los medios de comunicación, lo que disminuyó considerablemente el tiempo para difundir la información de un temblor. Sin embargo, aún en esos años, para construir un mapa de intensidades se seguía un proceso lento, instaurado desde los inicios de la RSN, en el que la recopilación de observaciones se hacía vía telefónica o a través de giras de campo. El mapa de intensidades resultante se obtenía días o semanas después de ocurrido el sismo fuerte y, generalmente, no se compartía con la población.

En este marco, la automatización de la localización, la difusión de sismos y la creación de herramientas para el cálculo de intensidades a partir de 2018, representan productos beneficiosos para la población, pues disminuyen la ansiedad de la espera para conocer las características de la sacudida de un sismo y contribuyen a crear conciencia rápidamente sobre la severidad del fenómeno (Bossu et al., 2018). Al mismo tiempo, brindar información rápida también es provechoso, pues facilita el compromiso de los usuarios para hacer sus reportes de intensidades (Bossu et al., 2018). En particular, el mapa promediado final basado en la información provista por usuarios comprometidos, es de gran utilidad para orientar tanto el trabajo de las instituciones que atienden emergencias, como para los medios de comunicación, ya que muestran cuán fuerte fue la sacudida del temblor y dan una idea de las zonas más afectadas por un sismo.

\section{Difusión de la información sismológica por diver- sas vías y con alto alcance}

La rápida publicación de las características de los sismos suele acompañarse de material educativo, el cual es difundido por una variedad de vías, como: la página web y las redes sociales. De esta manera, los usuarios tienen varias opciones para informarse rápidamente y desde plataformas que pueden alcanzar a miles de personas. Los medios usados por la RSN para comunicar información se revisan y mejoran cada año, con el fin de colocar más contenidos educativos y presentar la información sismológica en formas que sean más fáciles de comprender.

El sitio web actual de la RSN, se actualiza diariamente con la información de los últimos sismos y está diseñado para accederse fácilmente desde dispositivos móviles. Incluye mapas interactivos de sismos recientes, de fallas y de volcanes activos; además, información sobre sismos históricos, reportes volcánicos y resúmenes mensuales y anuales de la sismicidad desde 1976. Asimismo, la sección Educativos contiene preguntas frecuentes sobre las ciencias geológicas y un glosario sobre términos comunes que puede ser de gran beneficio para estudiantes y para docentes de primaria y secundaria. También, en esta sección, se incluyen sugerencias y medidas para enfrentar los terremotos con el fin de contribuir a la educación para la prevención de desastres.

Aunque en agosto de 2009 se crearon las primeras cuentas de la RSN en Facebook y Twitter, fue hasta enero de 2012 que se comenzó a brindar la información sismológica por esos medios. Rápidamente, las redes sociales empezaron a ser seguidas por miles de personas. En julio de 2020, la RSN posee alrededor de 400000 seguidores en Facebook, 170 000 en Twitter y 19000 en Instagram; por tanto, sus cuentas son de las más numerosas en seguidores de toda la UCR. Es así como, actualmente, cuando ocurren los sismos, los costarricenses no esperan a que la información sea anunciada en los noticieros en radio o televisión, como se hacía en años previos. En su lugar, los ciudadanos acceden a las redes sociales y buscan de inmediato los detalles y, además, comparten sus experiencias y sus observaciones en forma de reacciones y comentarios.

La principal motivación del uso de redes sociales en la RSN es proveer información y material educativo a la mayor cantidad posible de ciudadanos para, con esto, generar conciencia sobre el contexto geológico del país y preparar a la población para mitigar desastres. Lücke y Linkimer (2014) y Linkimer y Carvajal (2017) describieron en detalle los objetivos y contenidos de las redes sociales en la RSN, algunos de las cuales también son similares a los del Ovsicori (Vega, 2015). 
La RSN publica diariamente en Facebook y Twitter. El material que se comparte suele tratarse de textos cortos, fotografías, videos, enlaces a páginas web y animaciones. El contenido gira en torno a la educación y a la preparación para emergencias. Por ejemplo, se publica información sobre eventos sísmicos y volcánicos recientes a nivel nacional e internacional, notas educativas para conmemorar el aniversario de sismos o erupciones históricas en Costa Rica y el mundo, notas educativas sobre temas variados de las ciencias geológicas (figura 6), información sobre actividades, charlas de la RSN y visitas al campo. Adicionalmente, se realizan campañas con otros temas, como el papel de las mujeres en las ciencias.

Figura 6: Ejemplo de la campaña educativa sobre definiciones de términos comunes en geología y sismología. Esta publicación tuvo un alcance de casi 43 mil personas

\section{Epicentro}

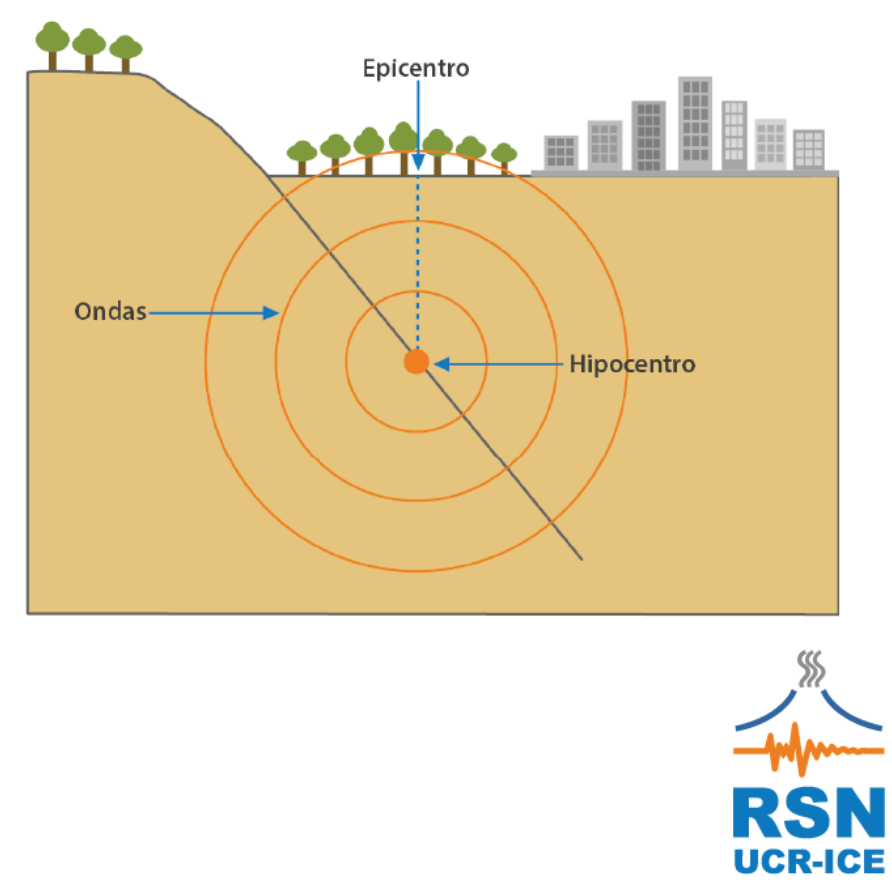

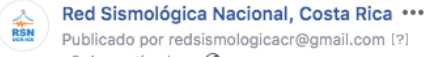
2 de septiembre 6

Hoy iniciamos una sección educativa que saldrá todos los lunes. Semanalmente se publicará una palabra del \#GlosarioRSN con términos relacionados a las áreas de geología, vulcanología, geomorfología y sismología.

Iniciaremos con la palabra epicentro, que se define como la primera zona de la superficie terrestre afectada por las ondas sísmicas y se sitúa directamente encima del hipocentro.

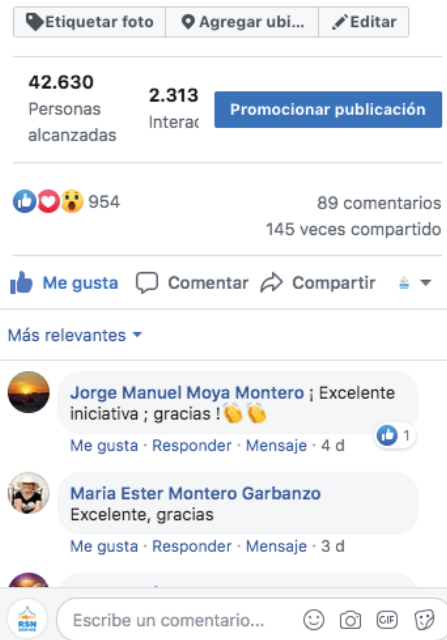

Fuente: Elaboración propia.

Se debe mencionar que debido al alto número de seguidores de las redes sociales y al alto alcance de las publicaciones, el material educativo que se comparte por estas redes impacta a muchas personas que tienen la posibilidad de aprender y familiarizarse con los fenómenos sismológicos.

Asimismo, otros mecanismos de comunicación usados por la RSN son: charlas en comunidades, visitas guiadas a la sede de las RSN y la atención a medios de comunicación masiva en prensa escrita, radio y televisión. Con el fin de acercar la ciencia a la población, también se han organizado, por ejemplo, actividades con miembros de comunidades indígenas y con niñas interesadas en las ciencias geológicas, charlas en comunidades afectadas por sismos recientes y visitas a medios de comunicación para capacitar a los periodistas en temas geocientíficos.

En el caso de la aplicación móvil RSN, esta ha evolucionado desde su lanzamiento en julio de 2015. La primera versión consistía únicamente en una plataforma sencilla con un resumen de las características básicas de los últimos sismos relevantes y un mapa de los eventos. Tanto la página web como esta aplicación móvil han sido renovadas en varias 
ocasiones; al respecto, las últimas versiones publicadas fueron en junio de 2017 y mayo de 2018, respectivamente.

Al notar la popularidad de la aplicación móvil en sus versiones iniciales y la anuencia de los usuarios de las redes sociales a reportar de forma masiva sus experiencias sobre los sismos, surgió la idea de potenciar y sistematizar la obtención de datos de intensidades. Así, nació la herramienta ¿Lo sentiste? (figura 3) disponible desde mayo de 2018 y el escenario de intensidades esperadas que fue concebido con el fin de tener un marco de referencia para la comparación de los mapas generados.

De acuerdo con López (2005), este tipo de iniciativas son muy beneficiosas ya que la ciudadanía, al sentirse involucrada, recibe el mejor estímulo para el aprendizaje y mejora su percepción ante las iniciativas públicas relacionadas con la ciencia. Asimismo, Asorey et al. (2017) señalan que la sociedad estará mejor preparada para hacerle frente a sus problemas en la medida en que las personas asuman un papel protagónico y se empoderen a través de las herramientas y de los conocimientos sobre los fenómenos que los rodean.

Entonces, la alta interacción de los usuarios al momento de ocurrir un sismo sentido, significa una gran oportunidad para recolectar información adicional y, así, determinar la intensidad, además de que posibilita direccionar a los usuarios a llenar el cuestionario del módulo ¿Lo sentiste? Asimismo, los contenidos con material educativo que se publican con frecuencia también alcanzan a miles de personas y promueven una alta interacción, lo que permite aclarar conceptos o responder preguntas comunes (figura 6).

Los resultados obtenidos evidencian, entonces, que la ciudadanía está dispuesta a asumir un rol activo en la generación de información cuando ocurre un sismo fuerte. Con el módulo ¿Lo sentiste?, las personas pueden aportar datos, obtener y visualizar la información básica sobre el sismo que acaban de percibir. Esta compenetración con el fenómeno sísmico fomenta su comprensión y refuerza las bases para el abordaje responsable de la amenaza sísmica. Al involucrar a la población en el proceso de reporte de intensidades sísmicas, se promueve una ciencia más interesante para los ciudadanos, que facilita la apropiación social de los contenidos.

Tanto la sociedad como la ciencia misma se han estado beneficiando mutuamente de este proceso, en donde los ciudadanos aprenden participando y se sensibilizan con los fenómenos que ocurren a su alrededor. Las herramientas presentadas han provisto un medio de comunicación más directo entre la RSN y la sociedad, y están contribuyendo a generar un ambiente de colaboración y participación social, en el que las geociencias se están acercado a una audiencia civil. Los resultados de décadas de esfuerzos en el mejoramiento de instrumentación, de sistemas de procesamiento y de investigaciones de las fuentes sísmicas del país, son ahora más accesibles a la población, lo cual es uno de los fines últimos de la ciencia.

\section{CONCLUSIONES}

Desde la RSN, se realiza un esfuerzo para informar rápidamente y educar a la ciudadanía a través de diversos mecanismos de comunicación que incluyen: una página web, una aplicación gratuita para teléfonos inteligentes, cuentas en redes sociales, charlas en comunidades, visitas guiadas a la sede de la RSN, la atención a los medios de comunicación y la elaboración de material educativo y campañas informativas. Esta tarea es muy importante en un país altamente sísmico como Costa Rica, en el que los movimientos telúricos son parte de la idiosincrasia de sus habitantes.

Entre las herramientas creadas por la RSN, existen dos dedicadas al cálculo rápido de la intensidad sísmica: el escenario de intensidades esperadas y el módulo ¿Lo sentiste? El primero se produce de forma automática con base en relaciones teóricas del decaimiento de la intensidad, y el segundo está basado en las observaciones aportadas por los ciudadanos. Estos mapas de intensidades y la información de las características básicas de los sismos se publican rutinariamente pocos minutos después de un sismo fuerte.

El módulo ¿Lo sentiste? es un ejemplo de ciencia ciudadana, en la cual se utiliza el aporte consciente y voluntario de los usuarios con observaciones de los sismos. De esta forma, se integra y realimenta 
permanentemente a la comunidad nacional y se pone al servicio de la sociedad la capacidad académica institucional con el fin de incidir en la calidad de vida de los habitantes.

Durante el tiempo analizado del módulo ¿Lo sentiste?, entre mayo de 2018 y julio de 2020, 17 sismos han tenido más de 500 reportes. El sismo con mayor participación, de casi 1300 personas, fue el terremoto de Armuelles de junio de 2019. Para este sismo, se construyó un mapa de intensidades que incluyó observaciones de todas las provincias de Costa Rica y dos de Panamá. Este mapa, basado en observaciones de los usuarios, muestra las mismas tendencias que el creado automáticamente, con valores máximos de VI y VII en la zona epicentral.

El uso efectivo de las redes sociales permite a los científicos y a las instituciones comunicarse con un gran número de personas. El sismo con mayor interacción del periodo analizado fue, también, el terremoto de Armuelles, el cual fue accedido en Facebook por 275 mil personas y comentado 9200 veces. Esto demuestra que las redes sociales de la RSN y otros mecanismos usados, como la aplicación móvil RSN, alcanzan un gran número de personas y representan una oportunidad para acercar las geociencias a una audiencia popular.

La difusión de la información sismológica por diversas vías, de forma rápida y con un alto alcance, como la aquí presentada, apunta a generar un ambiente en que la población esté informada, comprometida con el reporte de sus observaciones sobre los sismos y además educada en conceptos básicos de sismología. La educación, la comunicación efectiva y la alianza entre la academia y la sociedad propician una respuesta óptima ante la actividad sísmica, tarea fundamental en este país que se encuentra situado en una de las zonas más sísmicamente activas del planeta.

\section{AGRADECIMIENTOS}

Este trabajo es parte del proyecto de Acción Social ED-3005 Difusión de temas sismológicos de la UCR. La localización de sismos se realiza como parte del proyecto de investigación 113-B5-704 Vigilancia sísmica de Costa Rica también en la UCR. Adicionalmente a los recursos provistos por las vicerrecto- rías de Acción Social e Investigación, este proyecto ha empleado recursos económicos aportados a la RSN a través de la Ley de Emergencias 8488, que se gestiona en coordinación con la CNE. Con estos recursos, se logró financiar el diseño de la página web y de la aplicación para teléfonos móviles, el cual fue realizado en conjunto con Carlos Picado de la empresa IMACTUS.

Se agradece el apoyo de la Oficina de Divulgación e Información de la UCR. Los escenarios de intensidades fueron construidos en conjunto con Juan Luis Porras, de la RSN. La red de 167 estaciones sismológicas de la RSN se sostiene parcialmente a través de un convenio de cooperación científico y técnico entre la UCR y el ICE. El servicio de gran parte de las estaciones depende del trabajo de Luis Fernando Brenes y de Jean Paul Calvo. Asimismo, los siguientes asistentes de la RSN colaboraron en alguna medida con esta publicación: Luis Enrique Brenes, Silvia Carvajal, Mariana Mora y Justin Leiva.

\section{REFERENCIAS BIBLIOGRÁFICAS}

Allen, R. (2012). Transforming Earthquake Detection? Science, 335, 297-298. doi: 10.1126/science.1214650.

Arroyo, M., Linkimer, L. y Arroyo, I. (2020). Recuento de la sismicidad en Costa Rica durante el 2019. Revista Geológica de América Central, 62, 116-133. doi: 10.15517/rgac.v62i0.40639.

Asorey, H., Núñez, L.A., Peña-Rodríguez, J., SalgadoMeza, P., Sierra-Porta, D. y Suárez-Durán, M. (2017). Proyecto RACIMO: desarrollo de una propuesta en torno a uso de las TIC, e-ciencia ciudadana, cambio climático y ciencia de datos. Presentando en el congreso RedCLARA, Actas encuentro latinoamericano de e-Ciencia 2017, San José, Costa Rica.

Atkinson, G. y Wald, D. (2007). "Did You Feel It?" intensity data: A surprisingly good measure of earthquake ground motion. Seismological Research Letters, 78 (3), 362-368.

Blake, D., Johnston, D., Leonard, G., McLaren, L. y Becker, J. (2018). A Citizen Science Initiative to Understand Community Response to the Kaik ura Earthquake and Tsunami Warning in Petone and Eastbourne, WeIlington, Aotearoa/New Zealand. Bulletin of the Seismological Society of America, 108, 1807-1817. doi: https://doi.org/10.1785/0120170292. 
Ciencia ciudadana y herramientas de comunicación en la Red Sismológica Nacional de la Universidad de Costa Rica

Bossu, T., Roussel, F., Fallou, L., Landès, M., Steed, R., Mazet-Roux, G., Dupont, A., Frobert, L. y Petersen, L. (2018). LastQuake: From rapid information to global seismic risk reduction. International Journal of Disaster Risk Reduction, 28, 32-42. doi. https://doi. org/10.1016/j.ijdrr.2018.02.024

Crooks, A., Croitoru, A., Stefanidis, A. and Radzikowski, J. (2013). \#Earthquake: Twitter as a Distributed Sensor System. Transactions in GIS, 17, 124-147. doi:10.1111/ j.1467-9671.2012.01359.x.

Dengler, L. A. y Dewey, J. W. (1998). An Intensity Survey of Households Affected by the Northridge, California, Earthquake of 17 January, 1994. Bulletin of the Seismological Society of America, 88, 441-462.

Finazzi, F. (2020). Fulfilling the information need after an earthquake: statistical modelling of citizen science seismic reports for predicting earthquake parameters in near realtime. Journal of the Royal Statistical Society, 183, 857-882. doi:10.1111/rssa.12577

Gempa (2019). SeisComp 3 Real time data acquisition and processing [Software computacional]. Potsdam, Alemania: Gempa GmbH.

Gura, T. (2013). Citizen science: amateur experts. Nature, 496 (7444), 259-261.

Gutenberg, B. y Richter, C.F. (1942). Earthquake magnitude, intensity, energy, and acceleration. Bulletin of Seismological Society of America, 32, 163-191.

Gutenberg, B. y Richter, C.F. (1956). Earthquake magnitude, intensity, energy, and acceleration (second paper). Bulletin of Seismological Society of America, 46, 105145.

Linkimer, L. (2008a). Relationship between peak ground acceleration and modified Mercalli intensity in Costa Rica. Revista Geológica de América Central, 38, 81-95.

Linkimer, L. (2008b). Application of the Kriging method to draw the isoseismal maps of the significant 20022003 Costa Rican earthquakes. Revista Geológica de América Central, 38, 119-134.

Linkimer, L. y Alvarado, G. E. (2014). Distribución espacio-temporal de la sismicidad sentida en Costa Rica (1976-2013) en el marco histórico del 30 aniversario (1982-2012) de la Red Sismológica Nacional (RSN: UCR-ICE). Revista Geológica de América Central, Número Especial 30 aniversario, 45-71. doi: 10.15517/ rgac.v0i0.16569.

Linkimer, L. y Carvajal, S. (2017). El Uso de las Redes Sociales para aumentar el alcance de la Red Sismológica Nacional. Revista En torno a la Prevención, 19, 17-28.
Linkimer, L., Arroyo, I. G., Alvarado, G., Arroyo, M. y Bakkar, H. (2018). The National Seismological Network of Costa Rica (RSN): An Overview and Recent Developments. Seismological Research Letters, 89 (2A), 392-398.

López, J. A. (2005). Participación ciudadana y cultura científica. Arbor, 181 (715), 351-362.

Lücke., O. y Linkimer L. (2014): Redes Sociales y Sismología: educación y prevención en torno a amenazas sísmicas. Revista Reflexiones, 93 (2), 23-33.

Montero, W. (1989). Sismicidad histórica de Costa Rica. Geofísica Internacional, 28 (3), 531-559.

Montero, W. (2010). El Terremoto de Cartago del 4 de mayo de 1910: Aspectos sismológicos y neotectónicos. En G. Peraldo y B. Acevedo (Eds.), Efemérides de la destrucción de Cartago cien años después (19102010) (pp. 37-47). San José: Ediciones Perro Azul.

Montero, W. y Miyamura, S. (1981). Distribución de intensidades y estimación de los parámetros focales de los terremotos de Cartago de 1910, Costa Rica, América Central (Informe semanal Instituto Geográfico Nacional). San José: IGN.

Morales, L. D. (1986). Historia de la Sismología en Costa Rica. Revista de Filosofía de la Universidad de Costa Rica, 24, 93-104.

Ottemöller, L., Voss, P.H. y Havskov, J. (2020). Seismological Observatory Software: 30 Yr of SEISAN. Seismological Research Letters, 91 (3), 1846-1852. https://doi. org/10.1785/0220190313

Peraldo, G. y Montero, W. (1994). Los temblores del periodo colonial de Costa Rica. Cartago: Editorial Tecnológica de Costa Rica.

Porras, J. L. (2017). Atenuación sísmica en Costa Rica a partir de intensidades y Coda $Q$ (Tesis de licenciatura, Universidad de Costa Rica).

Porras, J.L., Linkimer, L., Arroyo, I.G., Arroyo, M., Taylor, M. y Fallas, C. (2019). La sismicidad del 2018 en Costa Rica. Revista Geológica de América Central, 60, 133144.

Vega, F. (2015). El volcán Turrialba y Ovsicori en redes sociales. Ambientico, 254, 24-37.

Wald, D.J., Quintoriano, V., Heaton, T.H. y Kanamori, H. (1999). Relationships between peak ground acceleration, peak ground velocity, and Modified Mercalli intensity in California. Earthquake Spectra, 15, 557-564.

Wald, D.J., Quintoriano, V., Heaton, T.H., Kanamori, H., Scrivner, C.W., y Worden, C.B. (1999). TriNet "Shake- 
Maps": Rapid generation of peak ground motion and intensity maps for earthquakes in southern California. Earthquake Spectra, 15, 537-555.

Wald, D.J., Quitoriano, V., Worden, C.B., Hopper, M. y Dewey, J. W. (2011). USGS "Did You Feel It?" Internet-based Macroseismic Intensity Maps. Annals of Geophysics, 54 (6), 688-707. doi: 10.4401/ag-5354.

Wood, H.O. y Newman, F. (1931). Modified Mercalli intensity of 1931. Bulletin of Seismological Society of America, 21, 277-283. 